\title{
High-frequency damping of plasmons in quasi-one-dimensional electron systems
}

\author{
B. Tanatar \\ Department of Physics, Bilkent University, Bilkent, 06533 Ankara, Turkey
}

(Received 7 November 1994)

\begin{abstract}
An asymptotic expression for the imaginary part of the dynamic dielectric function $\varepsilon_{2}(k, \omega)$ is studied for model quasi-one-dimensional (Q1D) electron systems. Derived within the two-particlehole pair excitation approximation, valid for all $k$ and high frequencies, $\varepsilon_{2}(k, \omega)$ is utilized to calculate the plasmon damping in a GaAs Q1D structure. An interpolation formula for the frequency dependence of the the dynamic local-field factor $G(k, \omega)$ is obtained.
\end{abstract}

\section{INTRODUCTION}

Quasi-one-dimensional (Q1D) electron systems as realized in semiconductor structures, in which the electrons are confined to moving freely only in one space dimension, have been a subject of much interest. With the advent of growth techniques, it has become possible to manufacture Q1D systems with many interesting experimental results. The main motivation for studying these low-dimensional systems comes from their technological potential such as high-speed electronic devices and quantum-wire lasers. Apart from the practical implications, electrons in Q1D structures offer an interesting many-body system for condensed-matter theories.

Collective excitations in an electron gas, may be studied as complex poles of the density-density response function $\chi(k, \omega)$ or as peaks of the dynamic structure factor $S(k, \omega)$. The density-density response function is quite generally expressed in terms of the proper polarizability $\Pi(k, \omega)$ and the interaction potential between the particles $V(k)$ :

$\chi(k, \omega)=\Pi(k, \omega) /[1-V(k) \Pi(k, \omega)]=\Pi(k, \omega) / \varepsilon(k, \omega)$,

where we have also defined the dynamic dielectric function $\varepsilon(k, \omega)$, the zeros of which describe the plasmons (collective excitations of an electron gas). In the randomphase approximation (RPA), the proper polarizability is approximated by the Lindhard function $\Pi^{0}$ (zerothorder diagram in perturbation theory). Experimental observation of collective excitations in Q1D electron systems came from far-infrared and magnetoresistance measurements ${ }^{1-3}$ and they are in accordance with theoretical predictions.

Landau damping is a decay mechanism for collective excitations in which the mode excites a single particlehole pair. In contrast to the $2 \mathrm{D}$ and $3 \mathrm{D}$ cases, the Q1D electron system does not exhibit Landau damping ${ }^{4}$ except on lines $k^{2} / 2 m \pm k k_{F} / m$, where $k_{F}$ is the Fermi momentum. Furthermore, the Q1D plasmons, due to their dispersion (within RPA), approach the Landau damping line $k^{2} / 2 m+k k_{F} / m$ only asymptotically. Thus, Q1D plasmons do not decay into a single particle-hole pair, and have a very long lifetime. A finite width (damping) of the collective excitations arises due to diagrams containing multiparticle-hole excitations in the intermediate states. Our aim is to study the damping of plasmons in Q1D electron systems originating from multipair excitations at high frequencies. Glick and Long ${ }^{5}$ have systematically investigated within the second-order perturbation theory the damping in a $3 \mathrm{D}$ electron gas, considering all two-particle-hole pair excitations. Extending this asymptotic approach, Holas and Singwi ${ }^{6}$ obtained results valid for all wave vectors $k$, and high frequencies $\omega \gg E_{F}$. There have been other approaches to investigating the $\varepsilon(k, \omega)$ in different regimes and approximation schemes. ${ }^{7,8}$

In this paper, we apply the analysis of Holas and Singwi ${ }^{6}$ to Q1D electron systems as they occur in semiconductor structures. For model interactions between electrons, we investigate the imaginary part of the (second-order) dielectric function $\varepsilon_{2}(k, \omega)$. Based on $\varepsilon_{2}(k, \omega)$, we estimate the plasmon damping at high frequencies, and study the dynamical local-field corrections by constructing a model that would be useful for further applications. Since the Q1D plasmons are undamped within the RPA, it is important to include the effects of multipair processes in order to obtain finite lifetime results at zero temperature.

The rest of this paper is organized as follows. In Sec. II we introduce some models for Q1D electron systems. The asymptotic form of $\varepsilon_{2}(k, \omega)$ in Q1D systems is presented in Sec. III. We use $\varepsilon_{2}(k, \omega)$ to calculate the high-frequency plasmon damping and to construct a frequency dependent local-field factor in Secs. IV and V, respectively. We conclude with discussion of our results and some remarks in Sec. VI.

\section{MODELS}

The first model we use in our calculation for the Q1D electron system is developed by $\mathrm{Hu}$ and $\mathrm{O}^{\prime}$ Connell, ${ }^{9}$ and is applicable to the experimental realizations of semiconducting systems. The charge carriers are assumed 
to be in a zero thickness $x-y$ plane with a harmonic confinement potential in the $y$ direction so that the subband energies are $\varepsilon_{n}=\Omega(n+1 / 2)$, where $\Omega$ describes the strength of the confining potential. The Coulomb interaction between particles in the lowest subband within this model is given by $V(k)=\left(2 e^{2} / \epsilon_{0}\right) F(k)$, where $F(k)=(1 / 2) \exp \left(b^{2} k^{2} / 4\right) K_{0}\left(b^{2} k^{2} / 4\right)$, in which $K_{0}(x)$ is the modified Bessel function and $\epsilon_{0}$ is the background dielectric constant. The characteristic length $b=1 /(m \Omega)^{1 / 2}$ is related to the confining potential. The large $k$ limit of the parabolic confinement model gives $F(k) \rightarrow(\pi / 2)^{1 / 2} /(b k)$.

As a second model, the Q1D structure is realized as a cylinder of radius $R$ with an infinite potential barrier. Gold and Ghazali ${ }^{10}$ have obtained an analytic expression for the Coulomb potential for this case using approximate wave functions

$$
\begin{aligned}
V(k)= & \left(\frac{2 e^{2}}{\epsilon_{0}}\right) \frac{36}{(k R)^{2}}\left[\frac{1}{10}-\frac{2}{3(k R)^{2}}+\frac{32}{2(k R)^{4}}\right. \\
& \left.-\frac{64}{(k R)^{4}} I_{3}(k R) K_{3}(k R)\right]
\end{aligned}
$$

For large $k$, the cylindrical confinement model gives $F(k) \rightarrow(36 / 10) /(k R)^{2}$. We note that the two models we consider differ in their large $k$ behavior. For long wavelengths, viz., $k \rightarrow 0$, both models behave as $\sim \ln (x k)$, where $x$ is some length parameter, depending on the Q1D model used. The weak logarithmic $k$ dependence, on the other hand, is a result of the effective Coulomb interaction between the charge carriers in a Q1D structure, and is essentially independent of the model describing them. ${ }^{11,12}$

\section{ASYMPTOTIC EXPRESSION FOR THE DIELECTRIC FUNCTION}

Employing the asymptotic formula of Holas and Singwi $^{6}$ for the imaginary part of the dielectric function corresponding to second-order diagrams with twopair excitations, we obtain for Q1D systems

$$
\begin{aligned}
\varepsilon_{2}(k, \omega)= & \left(\frac{4}{\pi}\right)^{4}\left(r_{s}^{3} / \pi\right) F(k)\left(\frac{k}{k_{F}}\right)^{4}\left(\frac{2 E_{F}}{\omega}\right)^{9 / 2} \\
& \times F^{2}(Q)[A(Q)-1]^{2}[1+\mathcal{O}(\eta)]
\end{aligned}
$$

where $A(Q)=[Q / V(Q)] d V / d Q, Q=(m \omega)^{1 / 2}$, and $E_{F}$ is the Fermi energy. In the above, $r_{s}$ is the electron gas parameter defined by $r_{s}=1 /\left(2 n a_{B}\right)$, where $n$ is the number density and $a_{B}=\epsilon_{0} /\left(m e^{2}\right)$ is the effective Bohr radius. The accuracy of the above expression (which is valid for any $k$ ) is of the order ${ }^{6} \eta$, defined as $\eta=$ $\left[\omega_{\text {asym }}(k) / \omega\right]^{1 / 2}$, where $\omega_{\text {asym }}(k)=\left(k^{2}+2 k_{F}^{2} / 3\right) /(2 m)$. Choosing $\eta \leq 0.7$ already results in better than $1 \%$ accuracy, as evidenced from exact Monte Carlo integration of the full integral..$^{-7}$ As shown by Holas and Singwi, ${ }^{6}$ the validity of the asymptotic formula for $\varepsilon_{2}(k, \omega)$ is also restricted to the region $\omega \gg \omega_{\mathrm{SPE}}(k)=\left(k^{2}+2 k k_{F}\right) /(2 m)$. We display various frequencies and frequency ranges in-

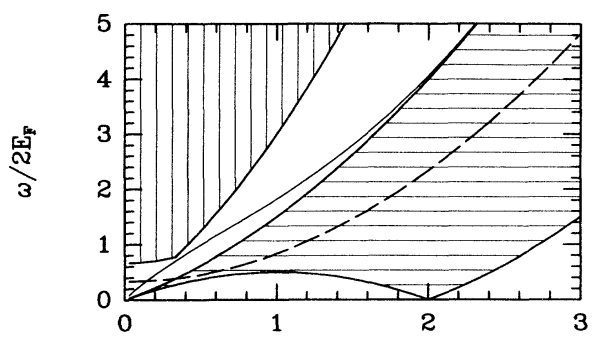

FIG. 1. Characteristic frequencies and frequency ranges for a Q1D electron system. Horizontally hatched region is the single-particle-hole region, of which the upper edge is $\omega_{\mathrm{SPE}}(k)$. Dashed line indicates $\omega_{\text {asym }}(k)$. Vertically hatched area is the estimated region of validity of the asymptotic form of $\varepsilon_{2}(k, \omega)$. Thin solid line gives the plasmon frequency, $\omega_{\mathrm{pl}}(k)$ for the cylindrical model at $R=a_{B}$, and $r_{s}=1$.

volved in this problem in Fig. 1. The vertically hatched area is the region of validity of the asymptotic form of $\varepsilon_{2}(k, \omega)$, calculated by $\omega \geq 2 \max \left[\omega_{\text {asym }}(k), \omega_{\mathrm{SPE}}(k)\right]$. Shown by the horizontally hatched region is the singleparticle-hole continuum. The upper edge of this region is the single-particle excitation line given by $\omega_{\mathrm{SPE}}(k)$. The dashed line indicates $\omega_{\text {asym }}(k)$. We also show, by the thin solid line, the plasmon frequency $\omega_{\mathrm{pl}}(k)$ for the cylindrical model with $R=a_{B}$, and $r_{s}=1$.

We note that the asymptotic form (i.e., $k \rightarrow 0, \omega \rightarrow \infty$ ) of the imaginary part of the dielectric function behaves as $\varepsilon_{2}(k, \omega) \sim|\ln (x k)| k^{4} \omega^{-11 / 2}$, or $|\ln (x k)| k^{4} \omega^{-13 / 2}$, for parabolic confinement or cylindrical models, respectively. Here we assumed that for any reasonable confinement model, the Q1D Coulomb potential $V(k \rightarrow 0) \sim$ $|\ln (x k)|$, where $x$ is some length parameter. These results for $\varepsilon_{2}(k, \omega)$ in Q1D are to be compared with the corresponding forms $k^{3} \omega^{-10 / 2}$ in $2 \mathrm{D}$ and $k^{2} \omega^{-11 / 2}$ in the 3D. The large $\omega$ dependence of the parabolic confinement model is similar to that of the $3 \mathrm{D}$ case, whereas the cylindrical model yields a much stronger $\omega$ dependence.

\section{PLASMON DAMPING}

The plasmon dispersion relation for Q1D electron systems is obtained in closed form within the $\mathrm{RPA}^{4,11,13}$

$$
\omega_{\mathrm{pl}}^{2}(k)=\frac{e^{u(k)} \omega_{+}^{2}-\omega_{-}^{2}}{e^{u(k)}-1},
$$

where $u(k)=(2 / \pi)(\pi / 4)^{2}\left(k / k_{F}\right) / F(k)$ and $\omega_{ \pm}=$ $2 E_{F}\left|\left(k / k_{F}\right)^{2} / 2 \pm\left(k / k_{F}\right)\right|$. The function $\omega_{\mathrm{pl}}(k)$ gives the plasmon energy (or frequency) for a given $k$, as the peak position in the energy-loss function $S(k, \omega)$. The width of the plasmon peak associated with a damping mechanism is also determined from the loss function, written for frequencies in the vicinity of $\omega_{\mathrm{pl}}(k)$. The full width at half maximum is given by

$$
\frac{\Gamma(k)}{2}=\left.\frac{\varepsilon_{2}(k, \omega)}{\varepsilon_{1}^{\prime}(k, \omega)}\right|_{\omega_{\mathrm{p} 1}(k)},
$$

where $\varepsilon_{1}^{\prime}(k, \omega)=\frac{\partial}{\partial \omega} \varepsilon_{1}(k, \omega)$, and more explicitly for Q1D 


$$
\begin{aligned}
& \text { systems, }{ }^{13} \\
& \begin{aligned}
\frac{\partial}{\partial \omega} \varepsilon_{1}(k, \omega)= & \frac{4\left(k / k_{F}\right)^{3}}{u(k)\left(2 E_{F}\right)} \\
& \times \frac{\left(\omega / 2 E_{F}\right)}{\left[\left(\omega / 2 E_{F}\right)^{2}-\tilde{\omega}_{-}^{2}\right]\left[\left(\omega / 2 E_{F}\right)^{2}-\tilde{\omega}_{+}^{2}\right]},
\end{aligned}
\end{aligned}
$$

in which $\tilde{\omega}_{ \pm}=\left|\left(k / k_{F}\right)^{2} / 2 \pm\left(k / k_{F}\right)\right|$. We have calculated the plasmon width $\Gamma(k)$, in our model Q1D systems using the RPA results for peak position $\omega_{\mathrm{pl}}(k)$, and $\varepsilon_{1}^{\prime}(k, \omega)$, while employing the asymptotic formula for $\varepsilon_{2}(k, \omega)$. In Fig. 2, we show $\Gamma(k)$ (in units of $2 E_{F}$ ) as a function of $k$ for the electron gas parameter $r_{s}=2$ and 3 (from top to bottom). The solid and dotted lines refer to the cylindrical wire and parabolic confinement models, respectively. We have used $R=a_{B}$ and $b=a_{B}$ in the calculations. The use of the asymptotic expression of $\varepsilon_{2}(k, \omega)$ in Eq. (5) requires that the ratio $\omega_{\mathrm{SPE}}(k) / \omega_{\mathrm{pl}}(k)$ be small. However, this quantity approaches unity as $k$ increases. The validity of our results for $\Gamma(k)$ also depends on the smallness of the parameter $\eta=\left[\omega_{\text {asym }}(k) / \omega_{\mathrm{pl}}(k)\right]^{1 / 2}$, which increases sharply for small $k$. Therefore, in Fig. 2, we have only plotted $\Gamma(k)$ for those values of $k$ that allow a meaningful estimate. We considered the region bounded by $\omega_{\mathrm{SPE}}(k) / \omega_{\mathrm{pl}}(k) \leq 0.5$ and $\eta \leq 0.5$. Similarly to the $2 \mathrm{D}$ case analyzed by Holas and Singwi, ${ }^{6}$ there appears a minimum $r_{s}$ below which the present method fails.

\section{LOCAL-FIELD CORRECTION}

As a second application of the asymptotic form of $\varepsilon_{2}(k, \omega)$ in Q1D systems, we consider the dynamical local-field corrections defined as

$$
G(k, \omega)=1 / Q^{0}(k, \omega)-1 / Q(k, \omega),
$$

where $Q^{0}(k, \omega)=-V(k) \Pi^{0}(k, \omega)$, and similarly for $Q(k, \omega)$. In particular, using Eq. (1) we have $Q(k, \omega)=$ $\varepsilon(k, \omega)-1$. The local-field corrections take the exchange and correlation effects into account, in an approximate way, neglected by the RPA. Since $\operatorname{Im} Q^{0}$ vanishes for frequencies $\omega>\omega_{\mathrm{SPE}}(k)$, we may write $\operatorname{Im} G(k, \omega)=$ $\varepsilon_{2}(\tilde{k}, \omega) /|Q(k, \omega)|^{2}$. In the large $\omega$ limit,

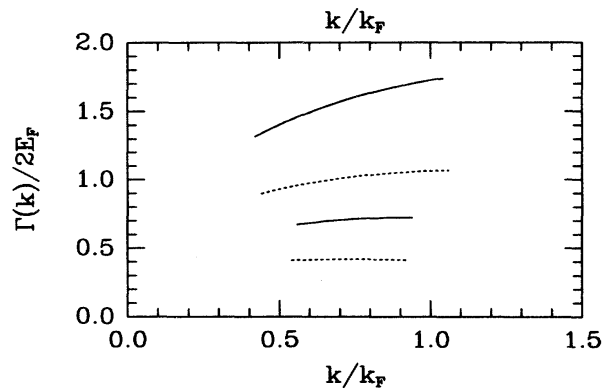

FIG. 2. Plasmon damping as a function of $k$, for cylindrical wire (solid lines) and parabolic confinement (dotted lines) models. Upper and lower curves are for $r_{s}=2$, and 3, respectively.

$$
Q(k, \omega)=-\left(\frac{\omega_{\mathrm{p}}^{2}(k)}{\omega^{2}}\right)\left[1+\mathcal{O}\left(\frac{1}{\omega^{2}}\right)\right],
$$

where $\omega_{\mathrm{p}}^{2}(k)=(4 / \pi)^{2} r_{s}\left(k / k_{F}\right)^{2} F(k)\left(2 E_{F}\right)^{2}$ is the longwavelength limit of plasmon dispersion relations. Note that $\omega_{\mathrm{p}}^{2}(k)$ may be obtained from the full RPA expression $\omega_{\mathrm{pl}}^{2}(k)$ by letting $u(k) \rightarrow 0$ (i.e., $k \rightarrow 0$ limit). Using now the asymptotic expression for $\varepsilon_{2}(k, \omega)$, the highfrequency limit of the imaginary part of the local-field factor becomes

$\operatorname{Im} G(k, \omega)=\frac{r_{s}}{\pi} \frac{1}{F(k)}\left\{\begin{array}{l}2 \pi /\left(b k_{F}\right)^{2}\left(2 E_{F} / \omega\right)^{3 / 2} \\ (108 / 10)^{2}\left(R k_{F}\right)^{-4}\left(2 E_{F} / \omega\right)^{5 / 2}\end{array}\right.$

for parabolic confinement and cylindrical models, respectively. We contrast this with the corresponding results of $\operatorname{Im} G \sim k \omega^{-1}$ and $\operatorname{Im} G \sim k^{2} \omega^{-3 / 2}$ in $2 \mathrm{D}$ and $3 \mathrm{D}$, respectively. We observe that the large $\omega$ behavior of $\operatorname{Im} G$ for the cylindrical Q1D model displays an unusual $\omega$ dependence. Since the $\operatorname{Im} G \sim \omega^{-3 / 2}$ case has been treated by Dabrowski ${ }^{14}$ in the context of $3 \mathrm{D}$ electron gas, we turn our attention to the $\operatorname{Im} G \sim \omega^{-5 / 2}$ behavior and develop a model for the local-field factor of a cylindrical quantum wire. The high-frequency $\operatorname{limit}$ of $\operatorname{Im} G$, combined with the small- $\omega$ behavior $\left(\lim _{\omega \rightarrow 0} \operatorname{Im} G \rightarrow \omega\right.$ in all space dimensions), may be used to construct a model $G(k, \omega)$ that incorporates these limits. We propose the form

$$
\operatorname{Im} G(k, \omega)=\frac{a(k)\left(\omega / 2 e_{F}\right)}{\left[1+b(k)\left(\omega / 2 E_{F}\right)^{2}\right]^{7 / 4}}
$$

in which $a(k)$ and $b(k)$ are wave vector and number density dependent parameters to be determined. The real and imaginary parts of the local-field factor are related by the Kramers-Krönig relations. This allows us to obtain $\lim _{\omega \rightarrow 0} \operatorname{Re} G(k, \omega)=G(k, 0)$, the static local-field factor, and $\lim _{\omega \rightarrow \infty} \operatorname{Re} G(k, \omega)=G(k, \infty)$. Comparison of Eq. (9) in the large- $\omega$ limit, and Eq. (10) yields $a(k)=$ $A b^{7 / 4}(k)$, where $A=\left(r_{s} / \pi\right)(108 / 10)^{2} /\left[\left(R k_{F}\right)^{4} F(k)\right]$. Considering the Kramers-Krönig relation at $\omega=0$,

$$
\begin{aligned}
G(k, 0)= & G(k, \infty) \\
& +\frac{2}{\pi} \int_{0}^{\infty} \frac{a(k)}{\left[1+b(k)\left(\omega / 2 E_{F}\right)^{2}\right]^{7 / 4}} d\left(\omega / 2 E_{F}\right),
\end{aligned}
$$

we finally get

$$
a(k)=A\left[\frac{G(k, 0)-G(k, \infty)}{A D}\right]^{7 / 5}
$$

and

$$
b(k)=\left[\frac{G(k, 0)-G(k, \infty)}{A D}\right]^{4 / 5},
$$

where the numerical constant $D$ is given by 


$$
D=\frac{2}{\pi} \int_{0}^{\infty} \frac{d x}{\left(1+x^{2}\right)^{7 / 4}} \simeq 0.55642 .
$$

This shows that a model of $G(k, \omega)$ may be constructed for a cylindrical quantum wire, provided the static localfield factors are known.

\section{DISCUSSION}

We have used the general formulation of Holas and Singwi ${ }^{6}$ to calculate the asymptotic form of the imaginary part of the dynamic dielectric function for Q1D systems. Considering the Q1D electron gas formed in confined structure in the lowest subband, we used the bare Coulomb interaction between the electrons. The lowest subband approximation is valid provided the higher subband energies are much larger than any other energy scales in the problem.

Bachlechner et $a .^{7}$ have investigated the plasmon damping in $3 \mathrm{D}$ and $2 \mathrm{D}$ electron gas within the secondorder perturbation theory, and showed the equivalence of different approaches that take the two-pair excitations into account. Carrying out a detailed Monte Carlo integration of the exact expression for $\varepsilon_{2}(k, \omega)$ which includes two-pair excitations, they showed that asymptotic results well represent the full $\varepsilon_{2}(k, \omega)$. We have not performed such a calculation for Q1D systems, but expect similar accuracy, provided that care is taken in handling the mathematical structure of the integrand. ${ }^{7}$

Recently, Hu and Das Sarma ${ }^{12}$ studied the many-body properties of Q1D electron systems using finite temperature perturbation theory techniques. They found that temperature and impurity scattering effects cause damping of the plasmons as $k$ increases. It is interesting to note that our calculated plasmon damping is of the same order for comparable electron densities and wire sizes, although the decay mechanisms in two cases are of different origin.

Our discussion in Sec. V shows that a parametrized model of a local-field factor may be constructed for Q1D electron systems consistent with the low- and highfrequency limits. The parameters of the model are the static local-field factors $G(k, 0)$ and $G(k, \infty)$, which, in turn, may be related to the correlation energy of the Q1D system similarly to the $2 \mathrm{D}$ and $3 \mathrm{D}$ electron gas. ${ }^{15}$ Presently, there are no parametrized expressions for the correlation energy of Q1D electron systems from which the static local-field factors can be deduced. A similar construction of $G(k, \omega)$ is also possible for different wire models.

Our results of plasmon damping and the dynamical local-field factor for widely employed models of Q1D electron systems could be used for further applications such as density-functional calculations.

\section{ACKNOWLEDGMENTS}

This work is partially supported by the Scientific and Technical Research Council of Turkey (TUBITAK).
${ }^{1}$ W. Hansen, M. Horst, J. P. Kotthaus, U. Merkt, Ch. Sikorkski, and K. Ploog, Phys. Rev. Lett. 58, 2586 (1987).

2 T. Demel, D. Heitmann, P. Grambow, and K. Ploog, Phys. Rev. B 38, 12732 (1988).

${ }^{3}$ A. R. Goñi, A. Pinczuk, J. S. Weiner, J. M. Calleja, B. S. Dennis, L. N. Pfeiffer, and K. W. West, Phys. Rev. Lett. 67, 3298 (1991).

${ }^{4}$ P. F. Williams and A. N. Bloch, Phys. Rev. B 10, 1097 (1974).

${ }^{5}$ A. J. Glick and W. F. Long, Phys. Rev. B 4, 3455 (1971).

${ }^{6}$ A. Holas and K. S. Singwi, Phys. Rev. B 40, 158 (1989).

${ }^{7}$ M. E. Bachlechner, W. Macke, H. M. Miesenböck, and A. Schinner, Physica B 168, 104 (1991); M. E. Bachlechner, H. M. Böhm, and A. Schinner, Phys. Lett. A 178, 186
(1993).

${ }^{8}$ W. Gasser, Z. Phys. B 57, 15 (1984); W. Gasser and Th. Fischer, Physica B 162, 43 (1990).

${ }^{9}$ G. Y. Hu and R. F. O'Connell, Phys. Rev. B 42, 1290 (1990).

10 A. Gold and A. Ghazali, Phys. Rev. B 41, 7626 (1990).

${ }^{11}$ Q. P. Li and S. Das Sarma, Phys. Rev. B 43, 11768 (1991).

12 B. Y.-K. Hu and S. Das Sarma, Phys. Rev. Lett. 68, 1750 (1992); Phys. Rev. B 48, 5469 (1993).

${ }^{13}$ G. Q. Hai, F. M. Peeters, J. T. Devreese, and L. Wendler, Phys. Rev. B 48, 12016 (1994).

${ }^{14}$ B. Dabrowski, Phys. Rev. B 34, 4989 (1986).

${ }^{15}$ N. Iwamoto, Phys. Rev. B 30, 3289 (1984). 